\title{
Controlling energy level offsets in organic/organic heterostructures using intramolecular polar bonds
}

\author{
Steffen Duhm, ${ }^{1, a)}$ Ingo Salzmann, ${ }^{1}$ Georg Heimel, ${ }^{1}$ Martin Oehzelt, ${ }^{2}$ Anja Haase, ${ }^{3}$ \\ Robert L. Johnson, ${ }^{4}$ Jürgen P. Rabe, ${ }^{1}$ and Norbert Koch, ${ }^{1, b)}$ \\ ${ }^{1}$ Institut für Physik, Humboldt-Universität zu Berlin, Newtonstr. 15, Berlin D-12489, Germany \\ ${ }^{2}$ Institut für Experimentalphysik, Johannes Kepler Universität Linz, Altenbergerstr. 69, Linz A-4040, Austria \\ ${ }^{3}$ Joanneum Research Forschungsgesellschaft mbH, Institute of Nanostructured Materials and Photonics, \\ Franz-Pichler Straße 30, Weiz A-8160, Austria \\ ${ }^{4}$ Institut für Experimentalphysik, Universität Hamburg, Luruper Chaussee 149, \\ Hamburg D-22761, Germany
}

(Received 4 December 2008; accepted 29 December 2008; published online 23 January 2009)

\begin{abstract}
The impact of intramolecular polar bonds (IPBs) on the energy level alignment in layered systems of rodlike conjugated molecules standing on the substrate was investigated for pentacene (PEN) and perfluoropentacene (PFP) on $\mathrm{SiO}_{2}$ using ultraviolet photoelectron spectroscopy. A remarkably large energy offset of $1.75 \mathrm{eV}$ was found between the highest occupied molecular orbital (HOMO) levels of PEN and PFP caused by IPBs at the surface of standing PFP layers. This large HOMO-level offset results in a narrow intermolecular energy gap of approximately $0.4 \mathrm{eV}$ at the interface between PEN and PFP layers. However, the absence of significant spatial overlap of PEN and PFP electron wave functions across the layers suppresses interlayer optical transitions. (C) 2009 American Institute of Physics. [DOI: 10.1063/1.3073046]
\end{abstract}

The energy level offsets in organic/organic interfaces are crucial parameters for the performance of optoelectronic devices based on organic compounds. ${ }^{1,2}$ Intensive research efforts are being carried out to understand the energy level alignment in organic heterostructures with the aim of achieving full control over interface energetics. ${ }^{3-6}$ Recently, the importance of intramolecular polar bonds (IPBs) on the electronic structure in organic/organic heterostructures was realized and it was demonstrated that IPBs can significantly impact the ionization energy (IE) of ordered molecular assemblies. $^{7,8}$ The IE is commonly believed to be a key parameter for energy level alignment. ${ }^{9,10}$ Pentacene (PEN) and its perfluorinated analog perfluoropentacene (PFP) are promising materials for use in organic electronics due to their high charge-carrier mobilities for holes (PEN) (Ref. 11) and electrons (PFP). ${ }^{12}$ Moreover, they are excellent model systems for investigating the impact of IPBs.

Ordered films of organic molecules without a net intrinsic molecular dipole moment can still exhibit sizable surface dipoles through the collective electrostatic effect of IPBs [see sketch for PEN and PFP in Fig. 1(a)], thus leading to a molecular-orientation dependence of the IE. ${ }^{7,8}$ For films formed by flat-lying PEN or PFP molecules (i.e., with the molecular planes oriented parallel to the substrate), the negatively charged $\pi$-electron cloud above each ring is exposed on the surface [Fig. 1(b)]. The resulting surface dipole layers are rather similar for flat-lying PEN and PFP and, therefore, the IEs of flat-lying PEN and PFP can be seen as their "intrinsic" IEs, which are not affected by IPBs. In contrast, in films of standing molecules the hydrogen atoms [carrying a slightly positive partial charge] (PEN) and the strongly electronegative fluorine atoms (PFP) are exposed at the surface. This results in surface dipoles of opposite sign that signifi-

\footnotetext{
${ }^{a}$ Present address: Graduate School of Advanced Integration Science, Chiba University, Chiba, Japan. Electronic mail: duhm@ restaff.chiba-u.jp.

${ }^{b)}$ Electronic mail: norbert.koch@physik.hu-berlin.de.
}

cantly impact the IE of the respective standing layers. Consequently, PEN and PFP exhibit a moderate difference in the IE $(\Delta \mathrm{IE})$ of $0.45 \mathrm{eV}$ for films of lying molecules, ${ }^{13}$ but a substantially increased $\Delta \mathrm{IE}$ of $1.85 \mathrm{eV}$ for films of standing molecules. ${ }^{8}$ Both molecules grow in an almost uprightstanding orientation in thin films on $\mathrm{SiO}_{2}$ substrates ${ }^{14-19}$ and this orientation was also reported for thin PFP films on top of PEN layers. ${ }^{19}$ Thus, the question arises whether IPBs permit the energy level offsets in organic/organic interfaces to be tuned, as was claimed for layered organic heterostructures of molecules with a defined orientation. ${ }^{7,8}$ Since the value of $\Delta \mathrm{IE}$ for layers of standing molecules $(1.85 \mathrm{eV}$; see above) is in the range of the optical energy gaps $\left(E_{\mathrm{opt}}\right)$ of both PEN $(1.85 \mathrm{eV})^{15,20}$ and PFP $(1.78 \mathrm{eV}){ }^{20}$ one might expect interlayer charge transfer (CT) between standing PEN and PFP molecules. In this study, we investigated (i) the mutual molecular orientation, (ii) the energy level offsets, and (iii) the optical properties of PEN-on-PFP and PFP-on-PEN layered structures using $\mathrm{x}$-ray diffraction (XRD), ultraviolet photoelectron spectroscopy (UPS), and UV-vis absorption spectroscopy. The experimental findings are supported by density functional theory (DFT) calculations.

PEN (Fluka) and PFP (Kanto Denka Kogyo Co., Ltd.) were vacuum sublimed (base pressure of $2 \times 10^{-9}$ mbar) at evaporation rates of about $1 \AA / \mathrm{min}$ using resistively heated pinhole sources. The film mass thickness was monitored with a quartz-crystal microbalance. (100) p-doped silicon wafers with a native oxide layer (Siegert Consulting, prime grade) were employed as substrates for UPS and XRD investigations; for UV-vis experiments polished fused silica (quartz) substrates (Präzisions Glas \& Optic $\mathrm{GmbH}$ ) were used. The $\mathrm{SiO}_{2}$ substrates were used as received; the quartz substrates were cleaned by subsequent sonication in acetone, isopropanol, and de-ionized water. Photoemission experiments were performed at the FLIPPER II end-station ${ }^{21}$ at HASYLAB (Hamburg, Germany). The spectra were recorded with 
(a)

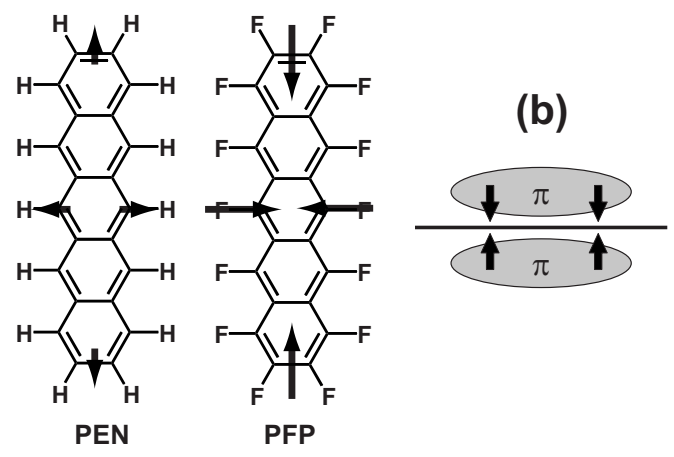

(c)

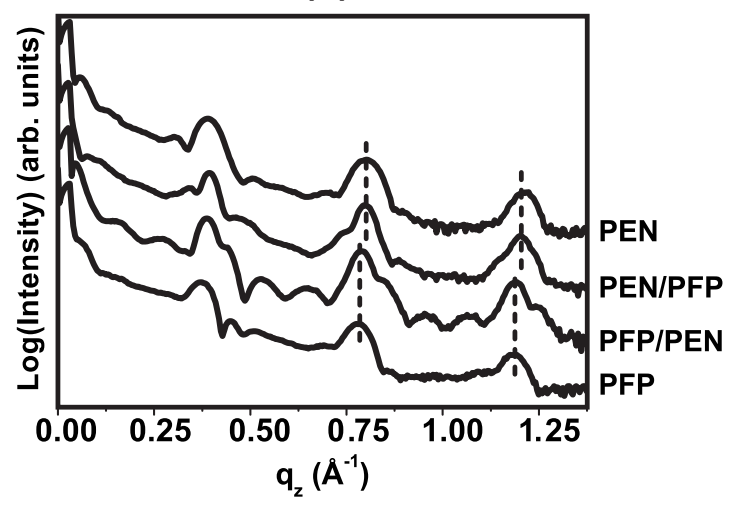

FIG. 1. (a) Molecular structures of PEN and PFP. The arrows indicate the direction and magnitude (dimensions not to scale) of intramolecular dipole moments originating from IPBs. (b) Schematic (lateral cut) through a planar conjugated molecule. (c) XRD scans (from top to bottom) of a $64 \AA$ thick PEN film on pristine $\mathrm{SiO}_{2}$ and on top of a $36 \AA$ PFP underlayer, of a $64 \AA$ thick PFP film on top of a $32 \AA$ PEN underlayer, and of the PFP film on pristine $\mathrm{SiO}_{2} . q_{z}$ is the momentum transfer perpendicular to the substrate plane.

a double-pass cylindrical mirror analyzer with an energy resolution of $150 \mathrm{meV}$. The photon energy was $22 \mathrm{eV}$. The samples were kept in the dark during synchrotron radiation exposure. The error of all given values of binding energies is estimated to be $\pm 0.10 \mathrm{eV}$. XRD measurements were performed at beamline W1 (Ref. 16) at HASYLAB under ambient conditions using an incident photon energy of $10.5 \mathrm{keV}$. Optical absorption measurements were performed using a Shimadzu UV-2101PC spectrometer with a resolution of $1 \mathrm{~nm}$ and (for the low energy region) a Lambda 900 spectrometer (PerkinElmer). All sample preparation steps and measurements were performed at room temperature. The DFT calculations on freestanding PEN and PFP monolayers were based on the thin film polymorphs of PEN (Ref. 16) and PFP (Ref. 19) on $\mathrm{SiO}_{2}$; computational details were the same as in Ref. 7 , differing only in the $k$-point grids of $5 \times 4(\mathrm{PEN})$ and $7 \times 3(\mathrm{PFP})$ used in the present study.

We have performed XRD measurements on pristine PEN and PFP films with coverages corresponding to nominally two standing layers of molecules and on layered structures of the two organic compounds; the results are depicted in Fig. 1(c). In all cases, we found growth in the PEN ${ }^{14,16,17}$ and $\mathrm{PFP}^{18,19}$ thin film phases. The layered PEN/PFP and PFP/ PEN films exhibited a superposition of the respective diffraction features of the pure films thus indicating an upright molecular orientation in all of the systems investigated.
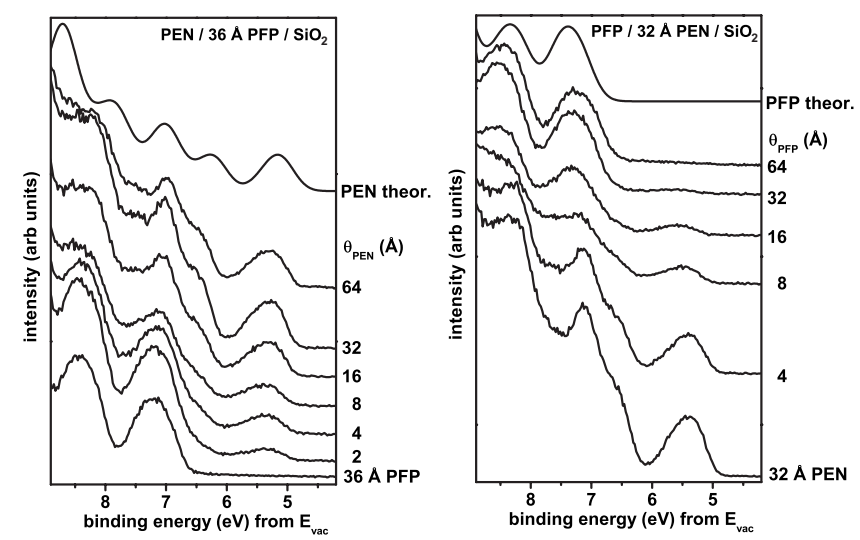

FIG. 2. Thickness dependent UPS spectra of $\mathrm{PEN}$ on $\mathrm{PFP} / \mathrm{SiO}_{2}$ (left) and PFP on $\mathrm{PEN} / \mathrm{SiO}_{2}$ (right). $\theta$ denotes the nominal layer thickness of the top material, $E_{\mathrm{vac}}$, the vacuum level. The spectra labeled "theor" correspond to the gausssian broadened DOS of a monolayer of the respective material. For PEN as for PFP the theoretical DOS was shifted to higher BEs by $1.15 \mathrm{eV}$ to account for the well-known shortcomings intrinsic to DFT.

The results of UPS investigations on the pristine and layered structures of PEN and PFP are presented in Fig. 2. The spectra of the pristine organic films exhibited an IE [onset of the highest occupied molecular orbital (HOMO) emission] of $\mathrm{IE}_{\mathrm{PFP}}=6.65 \mathrm{eV}$ and $\mathrm{IE}_{\mathrm{PEN}}=4.90 \mathrm{eV}$, reproducing recently reported values within the experimental error. ${ }^{8}$ The DFT-calculated density of states (DOS) of the respective monolayers fully captures the substantial difference between the IEs of films of standing PEN and PFP; the slight overestimate of $\Delta \mathrm{IE}_{\text {theor }}=2.09 \mathrm{eV}$ versus $\Delta \mathrm{IE}_{\text {expt }}=1.75 \mathrm{eV}$ is attributed to imperfections always present in real samples. Depositing the second compound onto the respective underlayer in a stepwise manner increasingly attenuated the intensity of the photoemission features of the underlying material, and concomitantly increased the intensity of the features of the top layer in both cases. No energy shifts or interactioninduced spectral features were observed, i.e., the spectra of the layered films were simply weighted superpositions of the pristine film spectra. For $\mathrm{PEN}$ on $\mathrm{PFP} / \mathrm{SiO}_{2}$ the onset of the PEN HOMO was at $4.85 \mathrm{eV}$ binding energy (BE) and for PFP on $\mathrm{PEN} / \mathrm{SiO}_{2}$ the onset of the PFP HOMO was at $6.60 \mathrm{eV}$ BE.

In Fig. 3(a) a schematic energy level diagram of the organic/organic interface is sketched. The energy positions of the HOMO levels are taken from the present UPS measurements. The position of the lowest unoccupied molecular orbital (LUMO) of PEN is estimated from the transport gap $\left(E_{\text {trans }}\right)$ of PEN $(2.20 \mathrm{eV}){ }^{10}$ Since no experimental data are available for $E_{\text {trans }}$ of PFP, it was estimated from the optical gap of PFP assuming a similar exciton BE as for PEN $\left(E_{\text {trans }}-E_{\text {opt }}=0.35 \mathrm{eV}\right)$, which yields a transport gap of $\sim 2.10 \mathrm{eV}$ for PFP. From the energy level diagram, a ground-state CT between PEN and PFP appears to be unlikely (and is indeed not observed). Although the difference in IE for PEN and PFP layers is in the range of the two (similar) optical energy gaps, the high BE edge of the PFP LUMO is still lower in energy than the low BE edge of the PEN HOMO. The UV-vis spectra of a layered structure (PFP/PEN) and the pure-film spectra (for comparison) are displayed in Fig. 3(b). The spectrum of the layered film can be perfectly reproduced as a superposition of the two purefilm spectra and, furthermore, no additional features were 
(a)

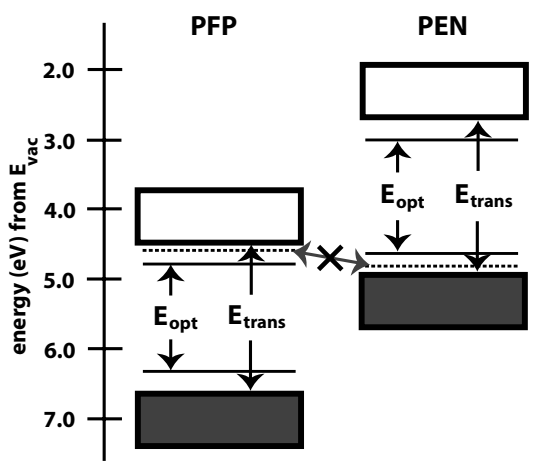

(b)

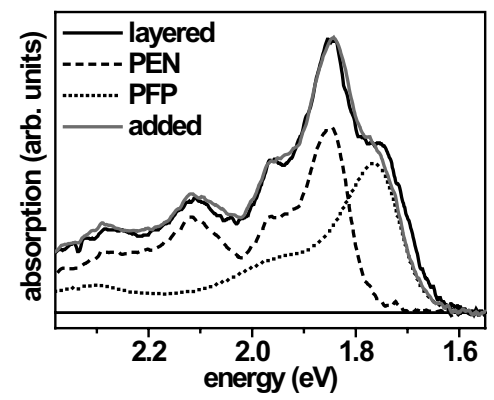

FIG. 3. (a) Energy level diagram of the PEN/PFP interface (standing molecules). The HOMO positions (gray bars) were measured with UPS, the LUMO positions (open bars) were estimated from the transport gaps $\left(E_{\text {trans }}\right)$, and the optical gaps $\left(E_{\text {opt }}\right)$ were taken from the absorption measurements. No intermolecular optical transition was detected from the HOMO of PEN to the LUMO of PFP (illustrated by the horizontal arrow). (b) UV-vis absorption spectra of pure PEN and PFP films, a layered (32 Å PFP/32 Å PEN) film, and a weighted superposition of the two pristine film spectra.

detected in the energy gap region (measured down to $0.40 \mathrm{eV}$, not shown), as indicated in Fig. 3(a). Importantly, no intermolecular optical transition was observed from the PEN HOMO into the PFP LUMO (expected onset around $0.40 \mathrm{eV}$ ); this was further evidenced by infrared absorption measurements (not shown) up to $0.50 \mathrm{eV}$. The absence of a noticeable CT absorption can be rationalized by the negligible spatial overlap of the wave functions (orthogonality) of PEN and PFP in the case of standing molecules in adjacent layers. One could assume that such an optical transition is allowed at the interface between layers of lying PEN and PFP molecules (in face-to-face orientation), however, $\Delta \mathrm{IE}$ and thus the energy level offset would be dramatically reduced in this scenario, leading to a significantly larger intermolecular energy gap. Compared to the standing case, where IPBs terminate the surface of the molecular layers and cause the large energy level offset, they cannot impact the energy level alignment at the interface between layers of face-toface lying molecules.

We have demonstrated that the position and strength of IPBs within a molecule allow the energy level offsets in organic heterostructures to be controlled. In the case of PEN and PFP, the IPBs increase the value of $\triangle \mathrm{IE}$ from $0.45 \mathrm{eV}$ for layers of lying molecules to $1.75 \mathrm{eV}$ for films of standing molecules. In layered heterostructures on $\mathrm{SiO}_{2}$, this results in a remarkably high HOMO-level offset of $1.75 \mathrm{eV}$ between vacuum-level aligned films of standing PEN and PFP (compared to an expected offset of $0.45 \mathrm{eV}$ for layers of lying molecules). It was demonstrated that-despite the resulting low energy offset between the PEN HOMO and the PFP LUMO levels-no ground-state CT occurs. Furthermore, no optical transition across the small intermolecular energy gap was observed and rationalized with the orthogonality of the respective electron wave functions. Interfaces with this type of energy level alignment may thus be useful as charge recombination zones in stacked organic devices.

The authors thank W. Caliebe (HASYLAB, Hamburg, Germany) for experimental support. N.K. acknowledges financial support by the Emmy-Noether-Program (DFG). G.H. is a Marie-Curie OIF fellow under the INSANE project (Contract No. 021511). This work was financially supported by the SFB 448 and the SPP 1355 (DFG).

${ }^{1}$ P. Peumans, A. Yakimov, and S. R. Forrest, J. Appl. Phys. 93, 3693 (2003).

${ }^{2}$ N. Koch, ChemPhysChem 8, 1438 (2007).

${ }^{3}$ H. Fukagawa, S. Kera, T. Kataoka, S. Hosoumi, Y. Watanabe, K. Kudo, and N. Ueno, Adv. Mater. (Weinheim, Ger.) 19, 665 (2007).

${ }^{4}$ W. Chen, H. Huang, S. Chen, X. Y. Gao, and A. T. S. Wee, J. Phys. Chem. C 112, 5036 (2008).

${ }^{5}$ S. Duhm, H. Glowatzki, J. P. Rabe, N. Koch, and R. L. Johnson, Appl. Phys. Lett. 90, 122113 (2007).

${ }^{6}$ W. Gao and A. Kahn, Appl. Phys. Lett. 82, 4815 (2003).

${ }^{7}$ S. Duhm, G. Heimel, I. Salzmann, H. Glowatzki, R. L. Johnson, A. Vollmer, J. P. Rabe, and N. Koch, Nature Mater. 7, 326 (2008).

${ }^{8}$ I. Salzmann, S. Duhm, G. Heimel, M. Oehzelt, R. Kniprath, R. L. Johnson, J. P. Rabe, and N. Koch, J. Am. Chem. Soc. 130, 12870 (2008).

${ }^{9}$ H. Ishii, K. Sugiyama, E. Ito, and K. Seki, Adv. Mater. (Weinheim, Ger.) 11, 605 (1999).

${ }^{10}$ A. Kahn, N. Koch, and W. Y. Gao, J. Polym. Sci., Part B: Polym. Phys. 41, 2529 (2003).

${ }^{11}$ S. Lee, B. Koo, J. Shin, E. Lee, H. Park, and H. Kim, Appl. Phys. Lett. 88, 162109 (2006).

${ }^{12}$ Y. Sakamoto, T. Suzuki, M. Kobayashi, Y. Gao, Y. Fukai, Y. Inoue, F. Sato, and S. Tokito, J. Am. Chem. Soc. 126, 8138 (2004).

${ }^{13}$ N. Koch, A. Vollmer, S. Duhm, Y. Sakamoto, and T. Suzuki, Adv. Mater. (Weinheim, Ger.) 19, 112 (2007).

${ }^{14}$ D. Nabok, P. Puschnig, C. Ambrosch-Draxl, O. Werzer, R. Resel, and D.-M. Smilgies, Phys. Rev. B 76, 235322 (2007).

${ }^{15}$ I. Salzmann, R. Opitz, S. Rogaschewski, J. P. Rabe, and N. Koch, Phys. Rev. B 75, 174108 (2007).

${ }^{16}$ S. Schiefer, M. Huth, A. Dobrinevski, and B. Nickel, J. Am. Chem. Soc. 129, 10316 (2007).

${ }^{17}$ H. Yoshida, K. Inaba, and N. Sato, Appl. Phys. Lett. 90, 181930 (2007).

${ }^{18}$ S. Kowarik, A. Gerlach, A. Hinderhofer, S. Milita, F. Borgatti, F. Zontone, T. Suzuki, F. Biscarini, and F. Schreiber, Phys. Status Solidi (RRL) 2, 120 (2008)

${ }^{19}$ I. Salzmann, S. Duhm, G. Heimel, J. P. Rabe, N. Koch, M. Oehzelt, Y. Sakamoto, and T. Suzuki, Langmuir 24, 7294 (2008).

${ }^{20}$ A. Hinderhofer, U. Heinemeyer, A. Gerlach, S. Kowarik, R. M. J. Jacobs, Y. Sakamoto, T. Suzuki, and F. Schreiber, J. Chem. Phys. 127, 194705 (2007).

${ }^{21}$ R. L. Johnson and J. Reichardt, Nucl. Instrum. Methods Phys. Res. 208, 791 (1983). 